\title{
Wideband Spectrum Compressed Blind Sensing without Reconstruction Based on Higher-order Moment
}

\author{
Chuan-Hai Jiao ${ }^{1,2}$ \\ ${ }^{1}$ State Key Laboratory of Complex Electromagnetic \\ Environment Effects on Electronics and Information \\ System \\ Luoyang 471003, China \\ ${ }^{2}$ Army Officer Academy \\ Hefei 230031, China \\ 凶jiao_chaunhai@126.com
}

\author{
Yong-Cheng Li \\ State Key Laboratory of Complex Electromagnetic \\ Environment Effects on Electronics and Information \\ System \\ Luoyang 471003, China \\ Rui Wang \\ Army Officer Academy \\ Hefei 230031, China
}

\begin{abstract}
To improve the detection performance and detection speed of wideband spectrum sensing algorithms with little priori knowledge, a scheme of cooperative wideband compressed blind sensing without reconstruction based on higher-order moment (CWCBS-HOM for short) is proposed. The test statistic is extracted from third-order moment of the compressed samples. The proposed method is blind in the sense since it need neither signal reconstruction, nor the prior knowledge of the primary user (PU) signal. Theoretical analysis and simulation results show that the proposed method can enhance the spectrum sensing capability with low computational complexity and high speed.
\end{abstract}

Keywords-Wideband spectrum sensing; Compressed sensing; Higher order moment; Blind sensing

\section{INTRODUCTION}

Spectrum sensing is one of the key technologies in cognitive radio (CR) [1] [2] [3]. However, with the increasing of communication rate and use of bandwidth, the high-speed sampling rate required is difficult to achieve under current technology level. The proposition and development of compressed sensing (CS), provide a new scheme for wideband spectrum sensing with low-speed sampling rate [4]. Classical reconstruction algorithms are applied to wideband spectrum sensing for single node [5] [6] [7], which can recovery signal with sub-Nyquist rate samples. To overcome the negative influence of wireless fading, many compressed wideband sensing methods in cooperative cognitive radio networks have been developed [8][9][10].

In the above studies, the prior knowledge of signal sparsity is assumed known. However, it is usually unknown in real scenario. Furthermore, many traditional wideband spectrum compressed sensing methods need restructure signal or its power spectral density (PSD) precisely, which may increase the computational complexity and detection time. In fact, the main task of spectrum sensing is not to restructure signal but to find spectrum holes. Then, we can use the compressed sampling measurements for spectrum sensing without signal reconstruction [11] [12].

In this work, we propose a method of cooperative wideband spectrum compressed blind sensing without reconstruction based on higher-order moment, hereinafter referred to as CWCBS - HOM method.

The remainder of this paper is organized as follows. In Sect. 2, we describe the cognitive radio networks and the signal model, and formulate our target problem. The CWCBS-HOM method is proposed and discussed in detail in Sect. 3. The simulation results and analysis for CWCBSHOM are shown in Sect. 4. Finally, the conclusions are drawn in Sect. 5.

\section{SYSTEM MODEL AND SIGNAL MODEL}

\section{A. System Model}

As shown in Fig.1, assume the cognitive radio network system includes one primary user (PU), one cognitive base station (CBS), and $L$ cognitive users (CUs). The PU and the CBS are far apart and the CUs are randomly distributed within the coverage radius of the CBS. In cooperative spectrum sensing, each CU sends its local information to the CBS via the common control channels while the CBS makes a fusion decision on the presence or absence of the PU signal.

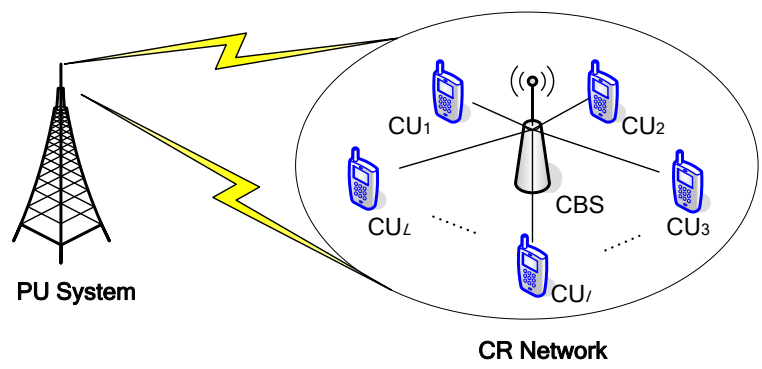

Figure 1. Scene model of central cooperative sensing 


\section{B. Signal Model}

When the $l$ th $(l=1,2, \cdots, L) \mathrm{CU}_{l}$ is sensing the channel, there are two hypotheses: hypothesis $H_{0}$ denotes the PU is inactive, and hypothesis $H_{1}$ denotes the $\mathrm{PU}$ is active. Therefore, the received signal compressed samples under the two hypotheses can be described as:

$$
\mathbf{y}_{l}= \begin{cases}\boldsymbol{\Phi} \mathbf{w}_{l}, & H_{0} \\ \mathbf{\Phi}\left(\mathbf{s}+\mathbf{w}_{l}\right), & H_{1}\end{cases}
$$

where $\quad \mathbf{w}_{l}=\left[w_{l}(1) w_{l}(2) \cdots w_{l}(N)\right]^{T} \quad$ denotes the Gaussian noise with zero mean and variance $\sigma_{w}^{2} . \mathbf{s} \in \mathbf{R}^{N}$ is the PU signal as a determined signal. $\mathbf{y}_{l}=\left[y_{l}(1) y_{l}(2) \cdots y_{l}(M)\right]^{T}$ is the $M \times 1$ dimension compressed sample vector received by $\mathrm{CU}_{l} . \boldsymbol{\Phi}$ is an $M \times N$ measurement matrix, which must satisfy restricted isometry property (RIP) condition [13][14]. We generate matrix $\boldsymbol{\Phi}$ by choosing the entries $\Phi_{i, j}$ as independent and identically distributed (i.i.d) sub-Gaussian random variables. We require that the distribution yields a matrix that is norm-preserving, which requires that $E\left(\Phi_{i, j}^{2}\right)=1 / M$.

\section{CWCBS-HOM METHOD}

The higher order statistics has different characteristic for the Gauss and non-Gauss signal. The essence is a measure of the deviation degree of Gauss, which can be used to detect the signal. Thus, the CWCBS - HOM method is proposed.

\section{A. Scheme Framework}

Fig.1 shows the scheme framework of CWCBS-HOM method. The CWCBS-HOM method contains two steps: CU local compressed sampling step and CBS fusion decision step.

In the first step, the $\mathrm{CU}_{l}(l=1,2, \cdots, L)$ samples the received signal $y_{l}(t)$ based on CS theory and calculate the estimated value of third-order moment $\bar{m}_{3 \mathbf{y}_{l}}$ using compressed sample vector $\mathbf{y}_{l}(l=1,2, \cdots, L)$. The local information is sent to the CBS via the common control channel. In the second step, the CBS gets the estimated thirdorder moment $\bar{m}_{3 \mathbf{y}}$ of joint observation vector $\mathbf{y}=\left[\mathbf{y}_{1} \mathbf{y}_{2} \cdots \mathbf{y}_{L}\right]$ as test statistic $J$ by means of the statistical averaging method. The joint decision threshold $\eta$ can be obtained by statistic characteristic of compressed sample vector and fixed false alarm probability (FAP). Finally, the decision result $u$ is given out.

\section{B. Method Description}

Under hypothesis $H_{0}$, there is only Gaussian noise, and the third-order moment of $\mathbf{y}_{l} \quad(l=1,2, \cdots, L)$ is $m_{3 \mathbf{y}_{l}}=m_{3 \mathbf{w}_{l}}=0$. Under hypothesis $H_{1}, m_{3 \mathbf{y}_{l}} \neq 0$. So, the decision rule in theory can be written as follows.

$$
\begin{cases}m_{3 \mathbf{y}_{l}}=0, & H_{0} \\ m_{3 \mathbf{y}_{l}} \neq 0, & H_{1}\end{cases}
$$

However, the formula above is not robust numerically. The reason is that we just can use the estimated value instead of the real value of HOM in practice. According to the definition of HOM, the estimated third-order moment of limited length compression measurements can be expressed as

$$
\bar{m}_{3 \mathbf{y}_{l}}=\frac{1}{M} \sum_{m=1}^{M} \mathbf{y}_{l}^{3}(m)
$$

Then, we get the test statistic $J$ by means of the statistical averaging method.

$$
J=\bar{m}_{3 \mathbf{y}}=\frac{1}{L} \sum_{l=1}^{L} \bar{m}_{3 \mathbf{y}_{l}}
$$

Combining (3) and (4), we obtain

$$
J=\frac{1}{L} \frac{1}{M} \sum_{l=1}^{L} \sum_{m=1}^{M} \mathbf{y}_{l}{ }^{3}(m)
$$

Similarly, the estimated third-order moment of Gaussian noise vector $\mathbf{W}$ can be described as

$$
\bar{m}_{3 \mathbf{w}}=\frac{1}{L} \frac{1}{M} \sum_{l=1}^{L} \sum_{m=1}^{M} \mathbf{w}_{l}{ }^{3}(m)
$$

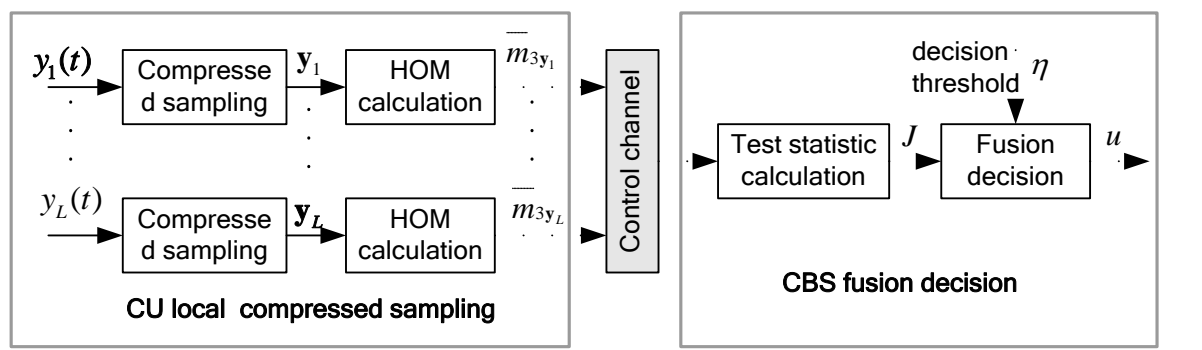

Figure 2. Scheme framework of CWCBS-HOM method 
Define the variance of $\bar{m}_{3 \mathbf{y}}$ as

$$
\sigma^{2}\left(\bar{m}_{3 \mathbf{y}}\right)=E\left[\left(\bar{m}_{3 \mathbf{y}}-m_{3 \mathbf{y}}\right)^{2}\right]
$$

Then, we have

$$
\bar{m}_{3 \mathbf{y}} \sim N\left(m_{3 \mathbf{y}}, \sigma^{2}\left(\bar{m}_{3 \mathbf{y}}\right)\right)
$$

Obviously,

$$
J \sim \begin{cases}N\left(0, \sigma^{2}\left(\bar{m}_{3 \mathrm{w}}\right)\right), & H_{0} \\ N\left(m_{3 \mathrm{y}}, \sigma^{2}\left(\bar{m}_{3 \mathrm{y}}\right)\right), & H_{1}\end{cases}
$$

The probability density function (PDF) of test statistic $J$ under hypothesis $H_{0}$ can be written as follows.

$$
p\left(J \mid H_{0}\right)=\frac{1}{\sqrt{2 \pi} \sigma\left(\bar{m}_{3 \mathrm{w}}\right)} \exp \left[-\frac{J^{2}}{2 \sigma^{2}\left(\bar{m}_{3 \mathrm{w}}\right)}\right]
$$

Comparing test statistic $J$ to joint decision threshold $\eta$, the decision rule for CWCBS-HOM method can be written as follows

$$
u= \begin{cases}0,|J| \leq \eta, & H_{0} \\ 1,|J|>\eta, & H_{1}\end{cases}
$$

where $\eta$ usually can be obtained by fixed FAP $P_{f}=\alpha . P_{f}$ can be expressed as

$$
P_{f}=P\left\{|J|>\eta \mid H_{0}\right\}=P\left\{J>\eta \mid H_{0}\right\}+P\left\{J<-\eta \mid H_{0}\right\}
$$

Combining (10) and (12), we have

$$
P_{f}=2 Q\left[\frac{\eta}{\sigma\left(\bar{m}_{3 \mathbf{w}}\right)}\right]=\alpha
$$

Thus, we have

$$
\eta=\sigma\left(\bar{m}_{3 \mathrm{w}}\right) \cdot Q^{-1}\left(\frac{\alpha}{2}\right)
$$

where $Q(\cdot)$ represents $\mathrm{Q}$ function, with $Q^{-1}(\cdot)$ as its inverse function. $\mathrm{Q}$ function is defined as

$$
Q(x)=\frac{1}{\sqrt{2 \pi}} \int_{x}^{\infty} \exp \left(-\frac{t^{2}}{2}\right) d t
$$

Obviously, the decision threshold $\eta$ is only associated with $\alpha$ and the variance $\sigma^{2}\left(\bar{m}_{3 \mathrm{w}}\right)$, but prior knowledge of PU signal and Gaussian noise variance $\sigma_{w}^{2}$.

\section{SiMULATION AND ANALYSIS}

Assume the PU signal is a wideband frequency hopping signal with hopping rate $300 \mathrm{H} / \mathrm{s}, 10$ hopping points, frequency range $20 \mathrm{MHz} 320 \mathrm{MHz}$. To investigate the performance of the proposed method under different compression ratio $M / N$ and different $\mathrm{SNR}$, We choose SNR range $[-30 \mathrm{~dB}, 5 \mathrm{~dB}]$, simulation step $1 \mathrm{~dB}, M / N=1 / 2,1 / 4,1 / 8$, $1 / 16$ and $1 / 32, N=1024$, CU number $L=10$, fixed FAP $\alpha=0.05$.

Fig. 3 shows the detection performance simulation results based on 1000 times Monte-Carlo simulation experiments.

From Fig.3, we can get the following views: 1) With compression ratio $M / N$ decreasing, the probability of detection reduces synchronously, especially at low SNR. 2) When SNR is above $-5 \mathrm{~dB}$, the probability of detection approximately equal 1 . In other words, the CWCBS-HOM method can detect the PU signal successfully.

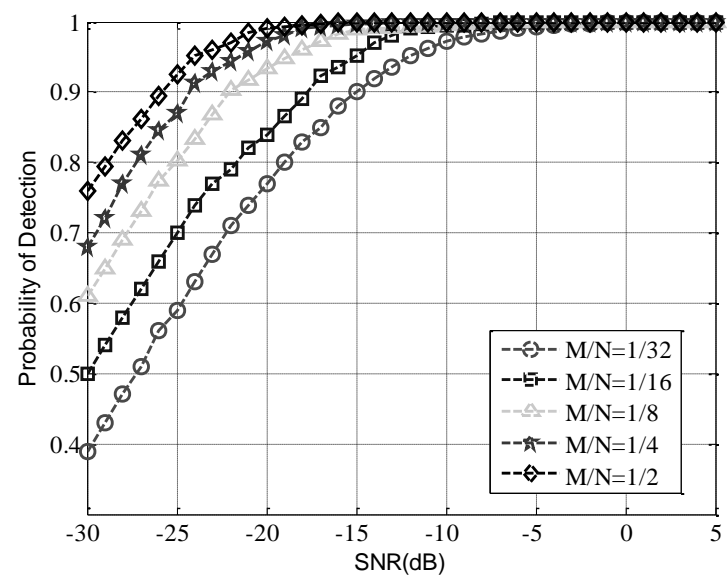

Figure 3. Probability of detection under different M/N

As comparison, the other two detection methods are simulated here. One is based on Roy's largest root test (RLRT) without reconstruction [15]. The other one is based on orthogonal matching pursuit (OMP) reconstruction algorithm [16]. The simulation parameters are set as follows: $N=1024, L=10, \alpha=0.05, M / N=1 / 4, \mathrm{SNR}=-20 \mathrm{~dB}$.

Fig. 4 shows the receiver operating characteristic (ROC) curves of the three methods without noise uncertainty(method- $0 \mathrm{~dB})$ and with $1 \mathrm{~dB}$ noise uncertainty(method $-1 \mathrm{~dB}$ ). 


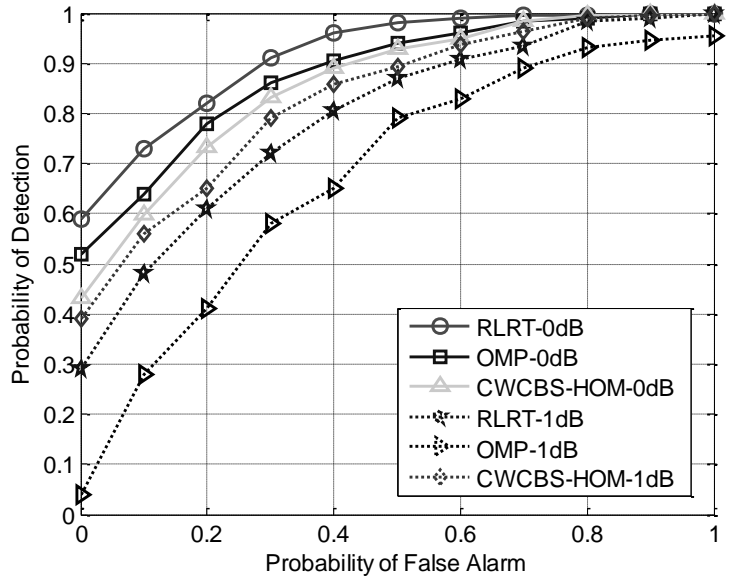

Figure 4. ROC curves of different methods

As shown in Fig.4, when there is no noise uncertainty, the RLRT method is better than OMP method and CWCBSHOM method. However, when there is noise fluctuation, CWCBS-HOM method is the best one among the three methods. Furthermore, CWCBS-HOM method is not sensitive to noise fluctuation. The other two methods are highly sensitive to the noise disturbance. The reason is that RLRT method need precise noise variance to get a relatively good detection performance. The OMP method has a large error in the reconstruction process because of the fluctuation of noise, so the detection performance is obviously decreased. In reality, due to the influence of the wireless environment, the noise is always fluctuating. Therefore, the proposed CWCBS-HOM method is more practical.

Fig. 5 shows the relationship between the detection time and the compression ratio $\mathrm{M} / \mathrm{N}$ of the above three methods. The simulation parameters are set to: $N=1024, L=10$, $\alpha=0.05, \mathrm{SNR}=-20 \mathrm{~dB}$.

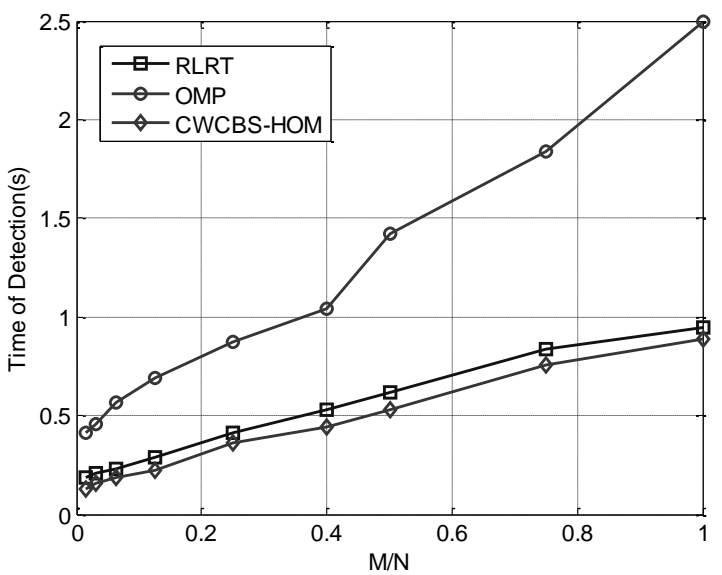

Figure 5. Detection time of different methods

It can be seen that the OMP method has the longest detection time. In contrast, the RLRT method and CWCBSHOM method have a faster detection speed. The complexity of the OMP reconstruction is much higher than that of the
RLRT method and CWCBS-HOM method without reconstruction.

\section{CONCLUSIONS}

In this paper, a method of cooperative broadband spectrum compressed blind sensing without reconstruction based on higher-order moment is proposed. In the method, the CU samples the received signal based on CS theory and calculate the estimated value of third-order moment using compressed sample vector directly. The CBS makes final decision by comparing the test statistic with the joint decision threshold.

The proposed method is a blind sensing scheme, since it does not require apriori knowledge of the PU signal or of the noise power. It can overcome the negative effect of low SNR and the presence of noise fluctuations. Simulation results show that compared with the other two methods, the proposed method has the advantages of low computational complexity, stable performance and so on. The method is suitable for the blind sensing of wideband signals in the central cooperative scenario when we have little priori knowledge. We will try to promote the proposed method to the blind sensing of the wideband random signals under the distributed cooperation scenario in the future.

\section{ACKNOWLEDGEMENT}

In this paper, the research was sponsored by State Key Laboratory of Complex Electromagnetic Environment Effects on Electronics and Information System Open Foundation (Project No. CEMEE2015Z0203B) and the Nature Science Foundation of Anhui Province (Project No. 1608085QF143).

\section{REFERENCES}

[1] Mitola III. J., Maguire G.O.: Cognitive radio: Making software radio more personal. IEEE Personal Communications, vol. 6, pp. 1318(1999)

[2] Haykin S.: Cognitive radio: brain-empowered wireless communications. IEEE Journal on Selected Areas in Communications, vol.3, pp. 201-220(2005)

[3] Haykin S., Thomson J., Reed H.: Spectrum sensing for cognitive radio. Proceedings of the IEEE, vol. 97, pp. 849-877 (2009)

[4] Tian Z., Giannakis G.B.: Compressed sensing for wideband cognitive radios. IEEE International Conference on Acoustics, Speech, and Signal Processing, pp. 1357-1360, Honolulu, USA (2007)

[5] Yen C.P., Tsai Y., Wang X.: Wideband spectrum sensing based on sub-Nyquist sampling. IEEE Transactions on Signal Processing, vol 61, pp. 3028-3040(2013)

[6] Pan L., Xiao S., Yuan X.: Wideband power spectrum sensing for cognitive radios based on sub-Nyquist sampling. Wireless Personal Communications, vol. 84, pp. 919-933 (2015)

[7] Khalaf T.A., Abdelsadek M.Y., Farrag M.: Compressed measurements based spectrum sensing for wideband cognitive radio systems. International Journal of Antennas \& Propagation, vol. 4, pp.1-7 (2015)

[8] Akyildz, I.F., Lo B.F., Balakrishnan R.: Cooperative spectrum sensing in cognitive radio networks: A survey. Physical Communication, vol.84, pp. 40-62 (2011)

[9] Arroyo-Valles R., Maleki S., Leus G.: Distributed wideband spectrum sensing for cognitive radio network. 2014 IEEE International 
Conference on Acoustics, Speech and Signal Processing, pp.72637267, Florence, Italy (2014)

[10] Grönroos S., Nybom K., Björkqvist J., et al.: Distributed spectrum sensing using low cost hardware. Journal of Signal Processing Systems, vol.83, pp.5-17(2016)

[11] Davenport M., Boufounos P., Wakin M., et al.: Signal processing with compressive measurements. IEEE Journal of Selected Topics in Signal Processing, vol.4, pp. 445-460 (2010)

[12] Abo-Zahhad M.M., Hussein A.I., Mohamed A.M.: Compressive sensing algorithms for signal processing applications: A survey. International Journal of Communications Network \& System Sciences, vol.8, pp.197-216 (2015)
[13] Baraniuk R., Davenport M., Devore R., et al.: A Simple Proof of the Restricted Isometry Property for Random Matrices. Constructive Approximation, vol.28, pp.253-263(2015)

[14] Eftekhari A., Han L.Y., Rozell C.J., et al.: The restricted isometry property for random block diagonal matrices. Applied \& Computational Harmonic Analysis, vol.38, pp.1-31(2015)

[15] Nadler B., Penna F., Garello R.: Performance of eigenvalue-based signal detectors with known and unknown noise level. Proceedings of the IEEE International Conference on Communications, pp.1-5, Kyoto, Japan (2011)

[16] Wang J., Kwon S., Li P., et al.: Recovery of sparse signals via generalized orthogonal matching pursuit: A new analysis. IEEE Transactions on Signal Processing, vol.64, pp. 1076-1089 (2016) 